\title{
MOSAIC+: tools to assist virtual restoration
}

\author{
Daniel Riccio \\ University of Naples Federico II \\ 80121 Campi Flegrei \\ Naples, Italy \\ daniel.riccio@unina.it
}

\author{
Sonia Caggiano \\ Master of Architecture and $\mathrm{PhD}$ \\ in Digital Painting Restoration \\ Salerno, Italy \\ soniacaggiano@yahoo.it
}

\author{
Riccardo Distasi, Michele Nappi \\ University of Salerno \\ 84084 Fisciano \\ Salerno, Italy \\ \{distasi,mnappi\}@unisa.it
}

\author{
Maria De Marsico \\ Sapienza University of Rome \\ Via Salaria, 113 \\ Rome, Italy \\ demarsico@di.uniroma1.it
}

\begin{abstract}
In many cases, virtual restoration is the only way to have an idea of the original appearance of an artwork. In particular, in the archeological field, it is useful to both assist and guide the operator in physical reconstruction, and to provide to the final visitor a complete vision of the artwork even though the original is damaged or lacks some parts. In this work, we propose a set of tools useful in two different restoration steps. The first one helps the expert to carry out reconstruction of fragmented artifacts in an easier and more effective way. The second provides a view of the artifact after virtually eliminating a craquelure.
\end{abstract}

Keywords-virtual restoration; mosaic fragmentation; painting craquelure; color indexing; shape indexing

\section{INTRODUCTION}

We start by the statement of the practical problem that we want to contribute to solve. It happens, either fortunately during archeological excavation campaign or unfortunately after destructive events, like earthquakes, to recover ruins originally covered by frescos. In the former case, the scene depicted is not known, in the latter pictures may exist, but the situation is not that better if fragmentation is dense. In both cases the automatic re-composition is very hard to carry out and requires patience and a lot of careful, slow work. Even worse, the inherent difficulty of the task is increased by its possibly extreme delicacy and by the required caution. The expert often faces a collection of painted fragments that crumble to dust if not handled with the utmost gentleness. On the contrary, reconstructing at least part of the original design would require much manipulation: correctly putting the pieces side by side requires an infinite number of repeated rotations, tentative alignments, and more operations on single pieces as well as batched of already dovetailed ones. In this scenario, the most time consuming action is to repeatedly explore the pool of available fragments to locate a possible candidate piece to join the portion of surface at hand. Each time a piece is touched or

DOI: 10.18293/DMS2015-049 moved it might break, or at least its edge might be further ground away, depending on the materials used to build and coat the old wall. At the end, when one luckily arrives at the end, it is the case that the recovered surface appears as cracked by missing pieces as well as by patchy edges. For the final visitor of the artwork, the artistic experience may be significantly improved by attenuating the visual effect of such irregularities.

This paper presents the advancements in the implementation of a set of tools formerly proposed as MultiObject Segmentation for Assisted Image reConstruction (MOSAIC) in [1]. MOSAIC+ also includes procedures to attenuate the visual effect of craquelure. Such tools are devised together with field experts to support the work of archaeologists and cultural heritage operators, when reconstructing fragmented (plain) artifacts. No information about the original appearance of the whole artwork is assumed to be available. To this aim, fragment images are inserted in a repository suitably indexed. The system provides the operator with complete workflow from photo-acquisition onwards. In the repository population phase, the fragments are photographed and their captured images are suitably processed and organized. The repository is indexed according to features such as color distribution, shape and texture. Images can be retrieved through query-by-example, using any fragment image as the key. If more results are available, as it is almost always the case, they are displayed to the user from the most to the least similar to the key. The operator can pick returned fragment images, rotate and translate them, and try to dovetail them to reconstruct the original picture, as when solving a puzzle. In most cases, holes will be present and the result will appear as highly fragmented, even in the virtual reconstruction. To further support the operator, as well as to provide an idea to the artwork visitors of its original appearance, once the reconstruction is completed a technique to attenuate craquelure is applied. Actually, this technique is also useful as a preprocessing step during population, before extracting shape and color information, when the fragments present inner craquelure. We applied our techniques on a number of 
simulations, and on the real use case of the reconstruction of a fresco from fragments found in the St. Trophimena church in Salerno (Italy).

\section{RELATED WORK}

MOSAIC+ includes an automated system for computeraided reconstruction of jigsaw puzzles. In algorithm literature, puzzles are grouped into two broad categories, that given the different characteristics need quite different approaches: in apictorial puzzles gather pieces of uniform surfaces that do not show figures, so that the only kind of information to be used to guide reconstruction is the fragment shape; in pictorial puzzles, texture and color information is available but, unlike most commercial puzzles, this does not necessarily imply that the solution image is known a priori. This the category of puzzles that restores usually handle.

The available literature offers several approaches to solve both types of jigsaw puzzles. Being the puzzle pictorial or apictorial does not significantly affect the computational complexity of automatic solution methods. In the first case, the classic paper by Freeman and Gardner, which was one of the earliest to tackle the problem of apictorial jigsaw puzzles, demonstrates that an exact algorithmic solution is NPcomplete: the computing time is super-polynomial with respect to the problem size [2]. The paper also suggests five fundamental puzzle properties: orientation (unknown a priori), connectivity (presence or absence of internal "holes"), perimeter shape (known/unknown a priori), uniqueness (does the problem admit only one solution?), radiality (topology of fragment juncture). The contours of the fragments are represented as chain codes, and code length is used as a heuristic for search space dimensionality reduction.

Some techniques can provide an approximate solution in a shorter time [3]. Applications are popular mostly in the fields of cultural heritage and ancient document reconstruction. A survey of literature is out of our scope, and in any case we do not tackle the problem of automatic solution, but rather that of relieving the expert by the burden of an extremely long trialand-error process and from the anxiety of manipulating critically fragile material. Nevertheless, it is worth mentioning some example system devoted to our same application field.

It is to notice that most techniques used in the cultural heritage field address the problem of pictorial puzzles, rather than apictorial. On the other hand, if the artifact to restore is a fresco, it is possible that it does not represent a natural scene but rather a set of repeated geometrical patterns. This latter circumstance may cause the lack of uniqueness of the combination of fragments, or of their automatically computed features at least.

The paper by Papaodysseus et al. facing the problem of reconstructing wall paintings [4] is paper particularly interesting for the present discussion. The focus is on the realworld issues that arise when dealing with a fresco: lack of information about the original content of the painting, possible non-uniqueness when geometric shapes are involved, and especially non-connectedness arising from the presence of very small fragments that are not available to the problem solver.
The technique for finding the correct correspondences deals with missing information using local curve matching.

Brown is among the authors of a semi-automatic system, used for reconstructing frescoes at the Akrotiri site in Thera, present Santorini in Greece [5]. The system carries out 3D data acquisition, but most results are obtained via 2D image feature extraction.

Brown is also among the experts dealing with the restoration of the Roman site in Tongeren (Atuatuca Tungrorum), the oldest town in Belgium. The site contained a number of artifacts that have been at least partially reconstructed, e.g., the Vrijthof Wall Decoration 1 [6]. The fragments were preliminarily acquired by an ad hoc 3D scanner. The shapes extracted by fragment image processing were matched by an ad-hoc software. Notwithstanding the efforts to refine image processing procedures to allow less expensive equipment, the total cost remained quite high. The obtained increased number of true matches is not that satisfactory, considering the actual numbers: 3 true matches were found manually, and in the same situation the system proposed 6103 tentative matches that became 17 true matches after human screening.

From results reported in literature, it is clear that better solutions can be obtained by fully exploiting all the possible available information. For instance, Chung et al. use color [7], while Sagiroglu and Ercil use texture [8]. It is to say that, in most cases, actual testing has been limited to problems involving a relatively small number of fragments. A completely different approach is presented by Nielsen et al. [9]. Fragment shape is neglected altogether. Rather, the method relies on features of the whole represented pictorial scene. The reported results for this technique show low error margins: the solution to a 320-fragment problem only had 23 pieces out of place-an error margin of $7.2 \%$ obtained by using only color and texture information. However, this approach implies to have at least a partial knowledge of the represented scene.

We can conclude by recognizing that the virtual reconstruction of pictorial fragments is an intrinsically hard problem. Approximate solutions as well as ones were the human-in-the-loop strategy is assumed are often all we can get. Several advanced image processing techniques are being incorporated in most recent systems. The most promising are based on local texture analysis, chrominance analysis and contour analysis on single fragments. When the original appearance is known or can be at least partially inferred, methods based on properties of the whole scene are expected to be quite powerful, and can provide further features to consider. Such techniques can produce representations that allow users to refine the solution progressively.

\section{CRAQUELURE}

In the artistic lexicon, craquelure is the pattern of fine and dense "cracks" on the surface of artworks. It can affect different materials, and it can be either due to an intentional characteristic of the production process, or be caused by defects in such process or more frequently by ageing. Though being a more general term, the most popular use refers to paintings, in particular those produced by tempera or oil, where it is causes 
mainly by ageing. In particular, in paintings on wood, it first appears following the direction of wood fibers. When referring to ceramics, it usually denotes a special manufacturing, and the term is usually modified as "crackle". Furthermore, it can appear on old ivory carvings, and on painted miniatures on ivory. Being a typical sign of ageing of the pictorial layer, the microscopic analysis of the craquelure (either natural or artificial, either deep or surface) is also used to determine the age and therefore the authenticity of paintings. We will slightly extend the term also to denote irregular and occasional cracking, especially on frescos (Fig. 1(b), cracking deriving from reconstruction with missing (small) parts, and virtual simulation of cracking (Fig. 1(e)). In particular, we will apply techniques to reduce the visual effect of craquelure, both on single fragments and after a virtual reconstruction of a fragmented fresco. Examples of craquelure are shown in Fig.1.

In order to hide the inter-fragment cracks in a reconstructed image, the craquelure pattern must first be detected, then corrected. The first methods for detection were only halfautomated and required human intervention [15], since the operator had to indicate a pixel in each connected component of the craquelure pattern, so that the system could locate a maximal connected region. Subsequent research produced methods that required less human intervention. The approach used by Giakoumis et al. [16] is based on mathematical morphology, as is the work by Spagnolo and Somma [17]. Both approaches require the operator to locate at least a small subset of the crack pattern, in order to train the system to detect cracks with either a light or a dark background. The solution proposed in this paper works with both types of craquelure without the help of a human operator, i.e. the technique adopted works notwithstanding the kind of craquelure.

Correction of the craquelure is performed by inpainting which is also used, e.g., to remove logos and similar superimposed marks from images, or to visually repair rips in the acquired version of a damaged image. There are several inpainting techniques. The more sophisticated ones aim at preserving the isophotes - curves of equal luminance in the underlying original image - or the luminance gradient in the neighborhood [18, 19, 20]. From the preserved isophote, a Laplacian smoothing or other interpolation can be used to extend the restoration to adjacent pixels. A simpler option is that of applying a spatial convolution mask to diffuse the surrounding pixels over the crack [21]. The tradeoff is between more accurate results in the case of isophotes preservation - or gradient-preserving techniques, vs. reduced computing time in the case of simple geometric processing.

\section{MOSAIC+ REPOSITORY}

Mosaic + can be classified among the toolsets for Jigsaw pictorial puzzle solving, where texture and color information is available but not the information about the original picture. Color and texture information is used together with shape information. As mentioned above, our proposal relies on a human-in-the-loop approach, i.e. it was expressly designed to support archaeologists and restorers facing fresco recomposition from fragments, and it does not implement an automatic approach. The aim is not to perform a completely automatic reconstruction, but rather to relieve the expert from most of the burden and anxiety implied by reordering fragile fragments and grouping them in similar clusters.

MOSAIC+ is composed of a number of modules, which implement different procedures. The first set of modules implements a procedure for image acquisition and processing. The result is a catalogued repository of the single fragments, which are clustered according to their texture/color and shape features. This preliminary grouping allows a quite quick answering to user queries, so that reconstruction is made easier, quicker and more effective. The interface module of the application provides a virtual workbench. Among the other actions, the user can virtually perform the actions that would have been performed on real fragments in a real reconstruction attempt, i.e., rotate, translate and search for similar fragments. In particular, a query engine allows searching the archive for relevant fragments, while the manipulation interface allows the user to manipulate them virtually to attempt recovery of the broken picture.

\section{A. Fragment image acquisition}

In the image acquisition phase, the physical fragments are laid in a white tray, whose bottom is covered by a dark foam to reduce reflexions. The distance among fragments must be sufficient to allow the following image segmentation to insulate the single pieces. The tray is placed inside a box for photographic acquisition, which is closed by a white curtain and bears two lateral spotlights. Close to the tray, a colorimeter is used by the operator to check for the need for automatic color corrections. The tray is then photographed. For this work, we used an 8-Mpixel Canon camera), orthogonally pointing it from a height of $90 \mathrm{~cm}$.

\section{B. Fragment image segmentation}

This operation aims at correctly separating each fragment appearing in a same image, so that individual features can be extracted from each of them. Segmentation entails two steps. The image is first binarized and turned into $\mathrm{B} / \mathrm{W}$ with no shades of gray. As can be expected, in our case no single threshold value is effective across all trays, unless some preprocessing occurs to enhance the image color separability. Too low values fail in separating pieces, while too high ones produce "holes" inside pieces. In extreme cases the piece may even come out as two separate fragments - an error that is quite hard to correct later. Therefore, the process of binarization that we carry out first entails pre-processing the raw image in order to amplify the difference between the brighter pixels (fragments) and the darker ones (the background foam is a dark shade of gray-almost black, but not quite). This preprocessing is described in detail in [1]. At the end of the segmentation process, specific information about the fragments found is computed, namely its area, its perimeter, and its orientation. The obtained binary connected component will be used as a mask to retrieve the fragment from the original image by a pixel-wise logical AND operation, in order to separately extract features from each fragment. Notice that, when putting fragments in the tray to acquire the tray image, fragments from very different groups can lay together, while fragments from the same group may lay in different trays. 


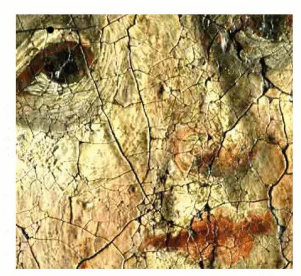

(a)

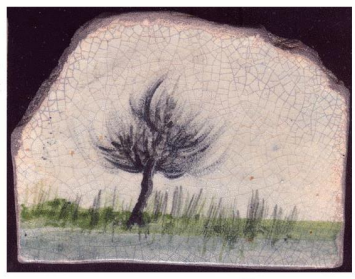

(d)

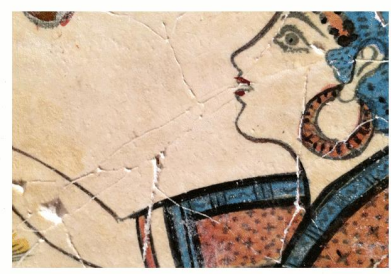

(b)

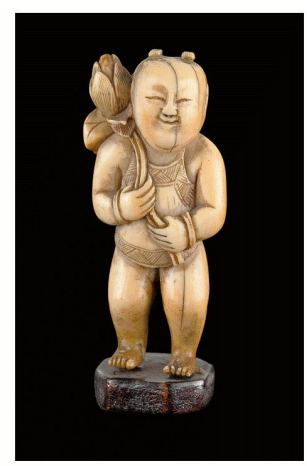

(c)

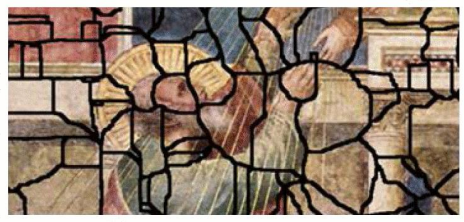

(e)

Figure 1. Examples of craquelure: (a) painting; (b) fresco; (c) ivory; (d) raku artwork; (e) virtual craquelure

\section{Fragment feature extraction}

Using the masks computed during segmentation, the module for feature extraction insulates the corresponding fragments, so that these can be individually indexed to allow a convenient successive retrieval. Indexing/retrieval are carried out according to the (basic) shape(s) depicted on the fragment, and to a spatiogram, which describes the spatial distribution of colors on the fragment surface [10]. The user can search the fragment catalogue by color, shape, or spatial color distribution, in order to retrieve fragments similar to a given "key" one. Details on feature extraction can be found in [1]

\section{Fragment search}

Extracted color information is stored by the fragment spatiogram; comparison is performed by related techniques. Shape information is represented in a more articulated way. Each shape on a fragment is represented as a triple

$$
\mathrm{S}=<\mathrm{v}, \omega, \mathrm{c}>
$$

where $v=(v 1, \ldots, v 7)$ is the vector of the first 7 central moments of the shape (see $[11,12])$, and $\omega$ and $c$, are the shape smoothness and mean color value, respectively. A fragment $F_{h}$ containing $s_{h}$ shapes is therefore characterized by $s_{h}$ such triples. We compare two shapes $\mathrm{S}_{1}=\mathrm{v}_{1}, \omega_{1}, \mathrm{c}_{1}>$ and $\mathrm{S}_{2}=\mathrm{v}_{2}$, $\omega_{2}, c_{2}>$, by computing their similarity as the normalized dot product of their moment vectors (i.e., the cosine of the angle between them), weighted by the product of their smoothness values:

$$
\operatorname{sim}\left(\boldsymbol{S}_{1}, \boldsymbol{S}_{2}\right)=\omega_{1} \omega_{2} \frac{V_{1} \boldsymbol{V}_{2}{ }^{T}}{\left|V_{1} \| V_{2}\right|}
$$

Since each fragment contains more shapes, the similarity between two fragments $F_{1}$ and $F_{2}$ is given by the maximum shape-to-shape similarity.
In the most common case, the query key is represented by a single shape $\mathrm{S}$ and the search goes through each fragment indexed in the database, looking for shapes with high values of similarity to $\mathrm{S}$. The similarity score assigned to a fragment is the maximum similarity score achieved by a shape it contains. Smaller shapes are discarded as not relevant.

\section{CRAQuelure attenuation}

\section{A. Craquelure detection}

Detecting and isolating craquelure traditionally required slightly different processing depending on the luminance of the cracks - either dark or light background. We adopt here a solution based on Mathematical Morphology (MM). Though MM is most often applied to digital images, it can be also employed on graphs, 3D surface meshes, and other spatial structures. Morphological operations on images entail the use of a suited structuring element, whose shape depends on the problem at hand, and which is used as a probe to draw conclusions on how this shape fits or misses the shapes in the image. Basic operations are erosion and dilation. Given an image $I$ and a structuring element $r$, the erosion of $I$ by $r$ is the locus of points reached by the center of $r$ when it moves inside $I$. The dilation of $I$ by $r$ is the locus of points covered by $r$ when its center moves inside $I$. Two further fundamental operations are derived, namely opening and closing. The opening (closing) of $I$ by $r$ is obtained by the erosion (dilation) of $I$ by $r$, followed by dilation (erosion) of the resulting image by $r$. Slightly different definitions hold when applying MM to either grayscale or color images. Details on morphological operators are out of the scope of this paper, but the reader can refer to the still extremely valid books [22] and [23]. In the case of MMbased processing, the most common solutions used the bottomhat operator for dark cracks and the top-hat operator for light cracks. Bottom-hat of an image $I$ and a structuring element $r$ is 
the difference between the closure of $I$ by $r$ and $I$, while top-hat is the difference between $I$ and its opening by $r$.

$$
\begin{aligned}
& \operatorname{Bhat}(I, r)=(I \bullet r)-I \\
& \operatorname{That}(I, r)=I-(I \circ r)
\end{aligned}
$$

The solution proposed in [24] to detect the crack pattern first transforms the image in grayscale, then uses the sum of bottom-hat and top-hat - namely, the difference between closure and opening. This operator is able to detect and isolate the cracks whether they have a dark or light background.

$$
\operatorname{Bhat}(I, r)+\operatorname{That}(I, r)=(I \bullet r)-(I \circ r) \quad \ldots
$$

The difference between closing and opening returns a grayscale image where the points of maximum and minimum luminance are made more evident than the rest of the image. However, lighter or darker brush traits provide false positives, therefore the image undergoes a thresholding operation to eliminate such false positives and create a map of areas with cracks. From the analysis of the histograms and the values of mean, standard deviation and modal value it comes out that pixels corresponding to these false positives have the gray values less than those that identify the true cracks. The threshold value is the sum of the mean value of the image pixels and the standard deviation. After thresholding, pixels that were discarded but have very similar values to the returned ones are added again, to obtain a more complete map. With the same goal, a dilation operation is finally performed. Differently from other approaches in literature, the solution we propose works indifferently with either light and dark craquelure, or with a mixture of them, without needing a human operator identifying the (possibly local) kind of craquelure in advance.

\section{B. Craquelure correction}

The resulting craquelure map is fed as input to the correction phase. Correction is performed by an inpainting method partly based on the Fast Marching Method illustrated in Telea's work [18]. The method fills the gaps with textures; however, in actual use cases the size of realistically correctable cracks seldom justifies texture creation, so a slightly different approach has been chosen: rather than creating a texture to fill the gap, the surrounding pixels are stretched by interpolation. This is similar to what an actual restorer does when repairing physical paintings - extending the remaining paint over the cracks by patient stretching.

\section{EXPERIMENTS AND RESULTS}

For our experiments we used both true fresco fragments found in the St. Trophimena church in Salerno (Italy), and a number of virtually cracked images.

A first consideration that is worth mentioning is that the measure of the structuring element used in MM depends on the thickness of the cracks, which in the real fragments are usually thinner than in the artificial images that we used. Therefore the structuring element is $3 \times 3$ for fresco fragments, and $9 \times 9$ for virtually cracked images. We denote by $I_{M M}$ the image resulting from applying the above mentioned morphological operations to the original image $I . I_{M M}(x, y)$ is the value of pixel in position $(x, y)$. The formula used to detect cracks and create map $M$ is:

$$
M(x, y)=\left\{\begin{array}{ccc}
1 & \text { if } & I_{M M}(x, y)>\left(\operatorname{mean}\left(I_{M M}\right)+\operatorname{std}\left(I_{M M}\right)\right) \ldots \\
0 & \text { otherwise }
\end{array}\right.
$$

By performing the experiments we also noticed that in large images (the artificial ones) the algorithm has difficulty in detecting cracks on the whole surface. We hypothesized that this depended on the fact that, being the images very large and variegated, the mean value of the entire image aggregates too much information and certain areas become unrepresentative. Therefore we decided to apply the algorithm to image patches. In other words, we divide the image into square patches of size $N x N$ and apply the algorithm to each of them, so rearranging the image of cracks as a collage of all the processed patches. In this way, each patch has its own average value, which is more representative and makes the detection of the cracks more accurate. The result, in fact, is far much acceptable.

We report the results of the following experiments: 1) craquelure detection with different dimensions of the patch and inpainting of the virtually cracked "Assunzione di san Giovanni Evangelista" by Giotto, Cappella Peruzzi cathedral of Santa Croce in Florence, about 1318-1322; 2) true fresco fragments before and after craquelure detection and inpainting; 3) a portion of the true reconstructed fresco before and after inpainting. The quality of results results can be appreciated visually. Fig. 2 shows the original image and the virtually cracked one of "Assunzione di San Giovanni Evangelista". Fig. 3-6 show the results of our procedure with patches of increasing size. It can be readily seen that as the patch size increases the inpainting procedure repairs the craquelure with less residual holes, and the result visually improves approaching the original appearance. This experiment is quite stressing due to the thickness of the craquelure pattern.

Fig. 7 and Fig. 8 show the images of two real fresco fragments (original image, craquelure map, inpainting result). As mentioned above, the best size of the patch to use depends on the resolution of the input. For fragments, all of which are relatively small, we used only 64x64patches.

Finally, Fig. 9 shows the image of a true part of the fresco rebuilt. Since the reconstructed part is bigger, we tested three possible choices: 64x64, 128x128 and 256x256. Fig. 10 and 11 show the results for $64 \times 64$ and 256x256 patches.

\section{CONCLUSIONS}

We presented MOSAIC+ (Multi-Object Segmentation for Assisted Image reConstruction), the evolving version of a system providing a set of tools to support the real as well as virtual reconstruction of fragmented pictorial artworks. We aim both at supporting the delicate work of experts, by facilitating the reconstruction of fragments, and at enhancing the experience of a visitor. The extraction of relevant features related to color and shape allows cataloging and indexing of the fragments, which support queries for similar pieces. 

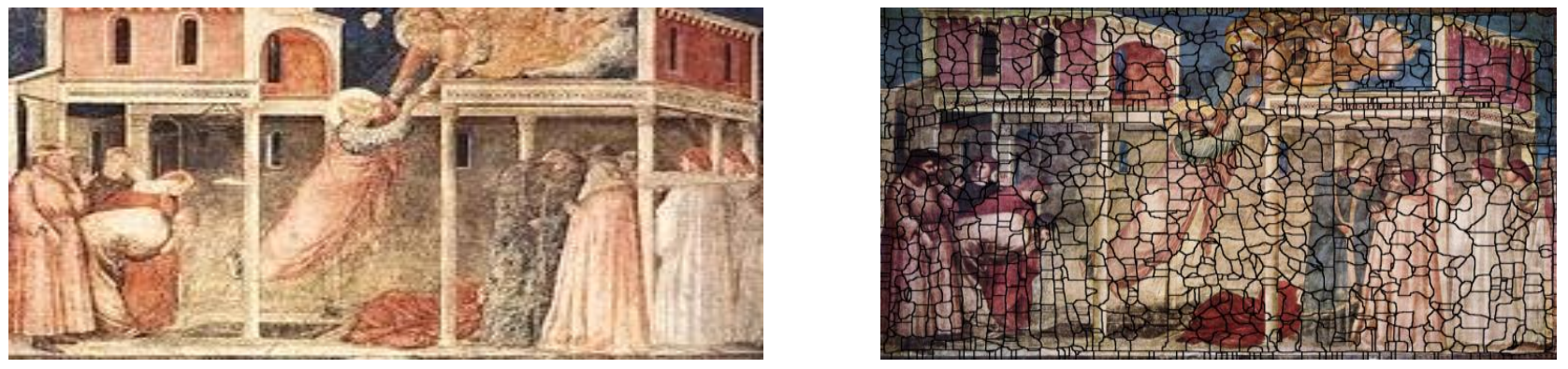

Figure 2. Examples of virtual craquelure: original fresco (left); virtual craquelure (right)

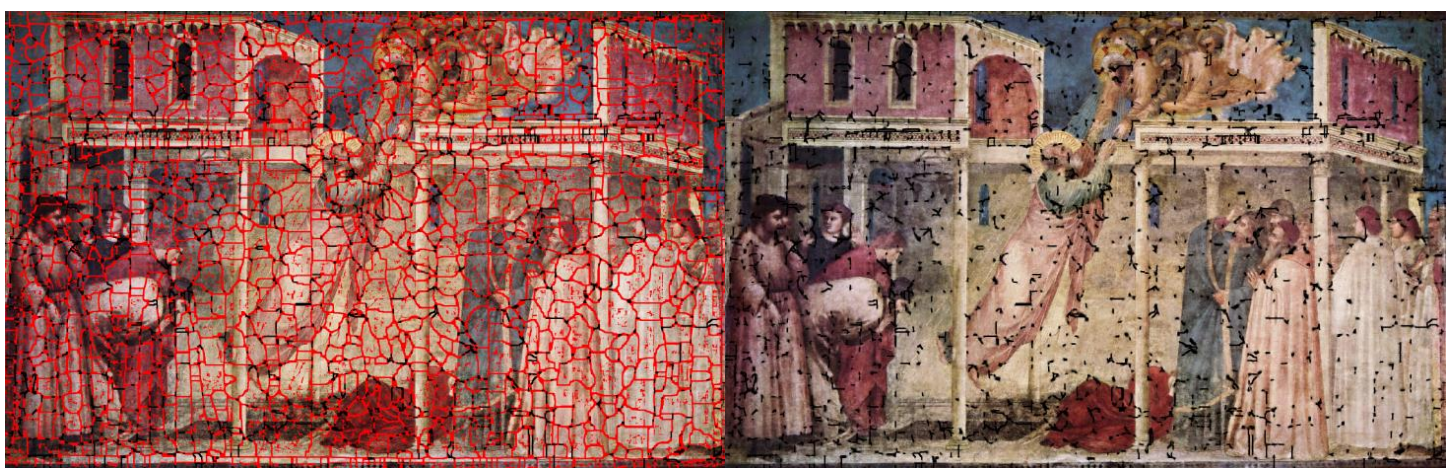

Figure 3. Results with $32 \times 32$ patches: (left) craquelure map; (right) inpainting

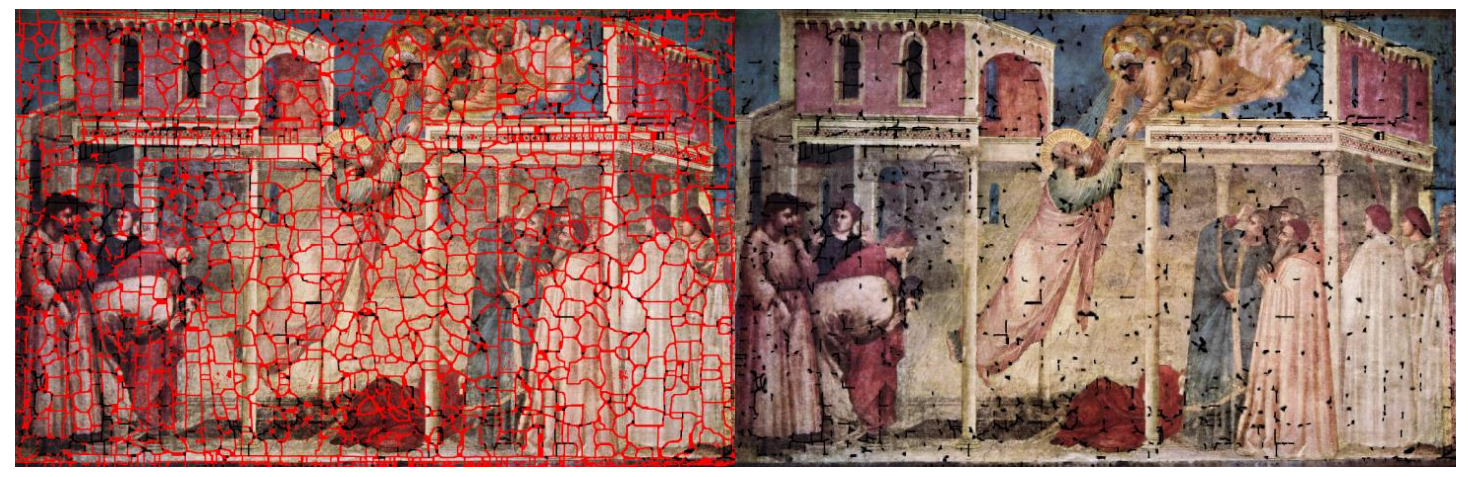

Figure 4. Results with $64 \times 64$ patches: (left) craquelure map; (right) inpainting

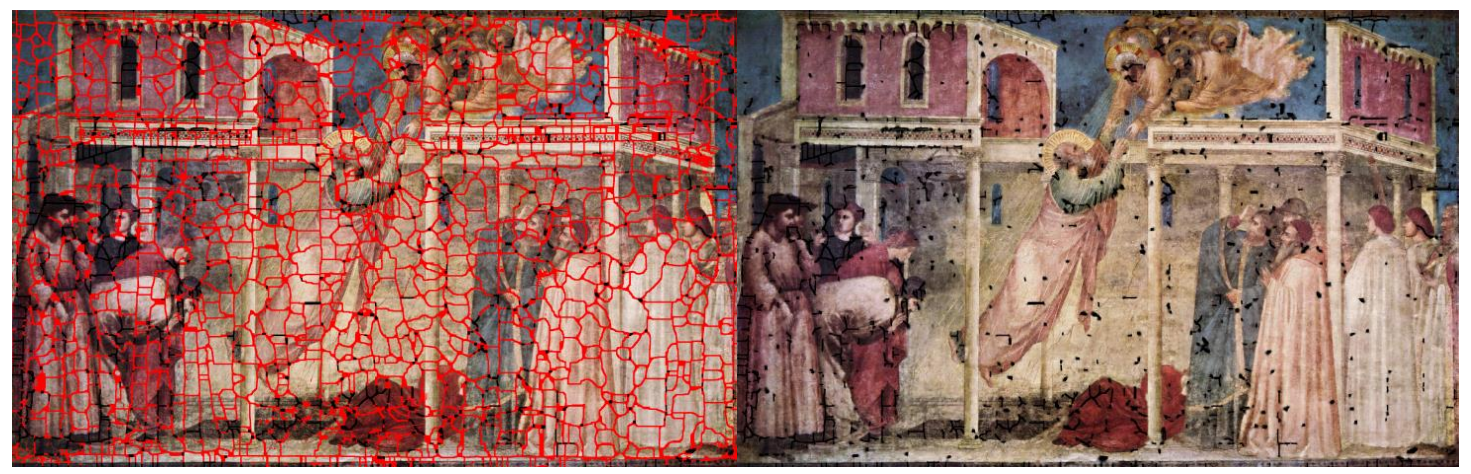

Figure 5. Results with $128 \times 128$ patches: (left) craquelure map; (right) inpainting 


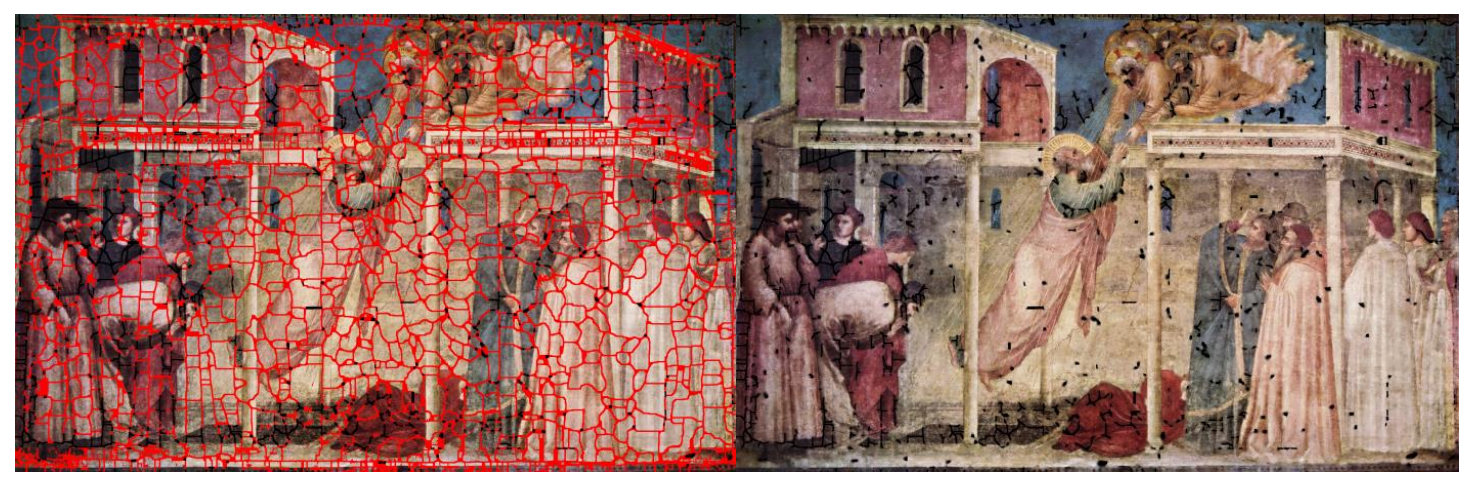

Figure 6. Results with $256 \times 256$ patches: (left) craquelure map; (right) inpainting

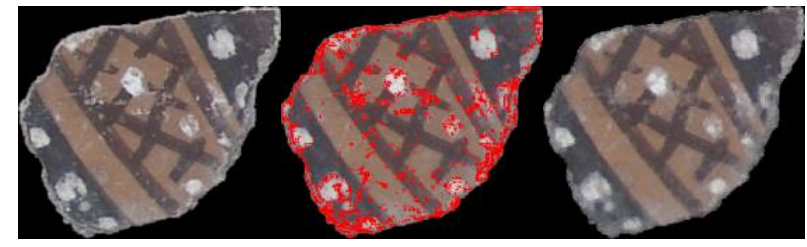

Figure 7. Fragment n. 00054-15 original (left), craquelure map (centre), inpainting (right)

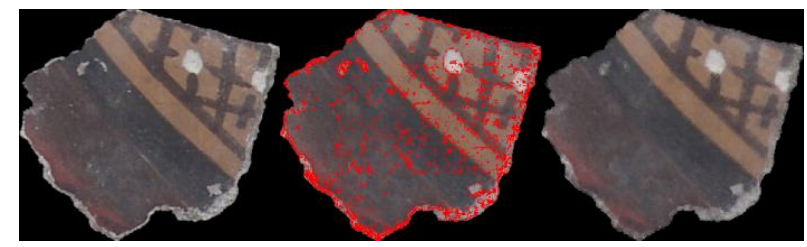

Figure 8. Fragment n. 00054-17 original (left), craquelure map (centre), inpainting (right)

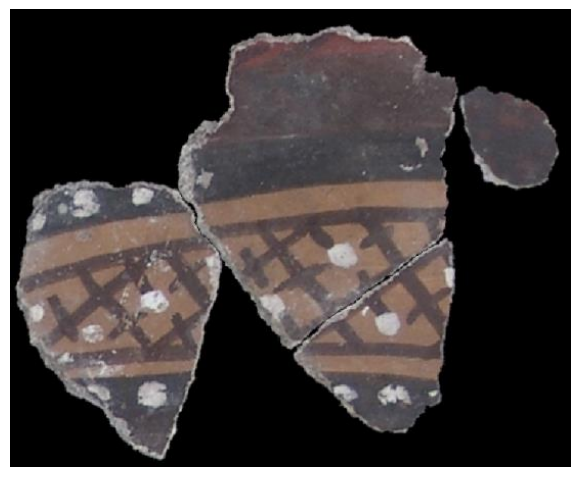

Figure 9. A portion of reconstructed fresco

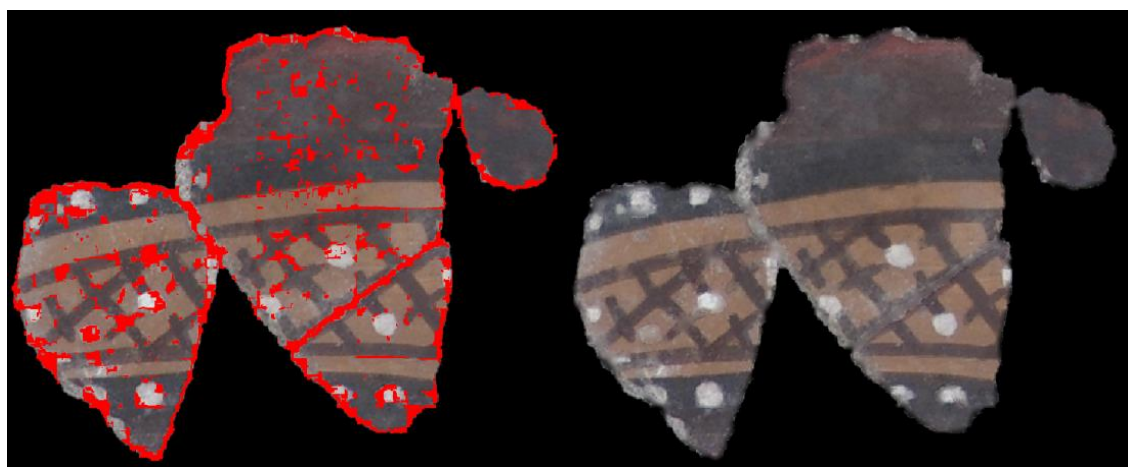

Figure 10. Virtual restoration of the part in Fig. 9 using 64x64 patches: (left) craquelure map and (right) inpainting 


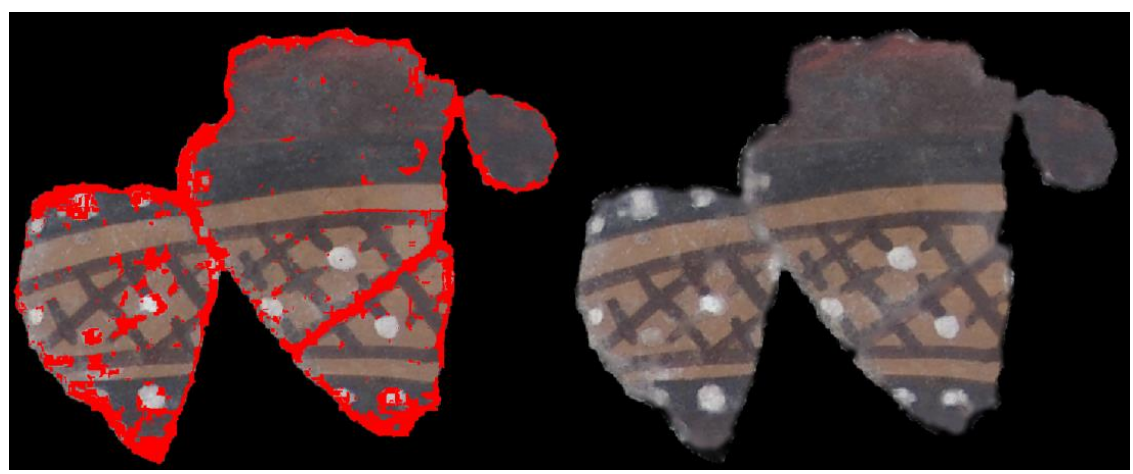

Figure 11. Virtual restoration of the part in Fig. 9 using 256x256 patches: (left) craquelure map and (right) inpainting

The results of the comparison with the stored virtual fragments are sorted by similarity to the query key, and this can speed up the manual reconstruction process significantly. We also experimented the effect obtained by virtually restoring craquelure, i.e., the presence of crack patterns due to age as well as to other negative factors. The system has been tested both via computer simulations and on a real case. The examples reported visually underline the quality that is possible to achieve in virtual restoration of the artwork appearance. This is deemed to improve the artistic experience of both experts and occasional visitors. Our future work will entail the implementation of better inpainting procedures, strategies for automatic setting of the right parameters according to the kind of artwork (size of the structuring pattern as well as of the image patches) and the addition of further tools.

\section{REFERENCES}

[1] S. Caggiano, M. De Marsico, R. Distasi, and D. Riccio. Multi-Object Segmentation for Assisted Image reconstruction. In Proceedings of 4-th International Conference on Pattern Recognition Applications and Methods - ICPRAM 2015, Vol. 2, pp. 100-107 (2015)

[2] H. Freeman, L. Garder, "Apictorial jigsaw puzzles: The computer solution of a problem in pattern recognition." IEEE Transactions on Electronic Computers 2(EC-13), 118-127 (1964)

[3] T.S. Cho, S. Avidan, W.T. Freeman, "A probabilistic image jigsaw puzzle solver.” In: CVPR. pp. 183-190. IEEE (2010), http://dblp.unitrier.de/db/conf/cvpr/cvpr2010.html\#ChoAF10

[4] C. Papaodysseus, T. Panagopoulos, M. Exarhos, "Contour-shape based reconstruction of fragmented, 1600 bc wall paintings." IEEE Transactions on Signal Processing 6(50), 1277-1288 (2002)

[5] B. Brown, C. Toler-Franklin, D. Nehab, M. Burns, D. Dobkin, A. Vlachopoulos, C. Doumas, S. Rusinkiewicz, T. Weyrich, "A system for high-volume acquisition and matching of fresco fragments: Reassembling Theran wall paintings." ACM Transactions on Graphics (Proc. SIGGRAPH) 27(3), 1-10 (2008)

[6] B. Brown, L. Laken, P. Dutrè, L.V. Gool, S. Rusinkiewicz, T. Weyrich, "Tools for virtual reassembly of fresco fragments." In: Proceedings of the 7th International Conference on Science and Technology in Archaeology and Conservations. pp. 1-10. SCITEPRESS (2010)

[7] M.G. Chung, M. Fleck, D. Forsyth, "Jigsaw puzzle solver using shape and color." In: Proceedings of the 4th International Conference on Signal Processing (ICSP '98). vol. 2, pp. 877-880 (1998)

[8] M. Sagiroglu, A. Ercil, "A texture based matching approach for automated assembly of puzzles." In: Proceedings of the 18th International Conference on Pattern Recognition (ICPR '06). pp. 10361041 (2006)
[9] T.R Nielsen, P. Drewsen, K. Hansen, "Solving jigsaw puzzles using image features". Pattern Recognition Letters 14(29), 1924-1933 (2008)

[10] S.T. Birchfield,S. Rangarajan, "Spatiograms versus histograms for region-based tracking." In: Proceedings of the IEEE Conference on Computer Vision and Pattern Recognition (CVPR). pp. 1158-1163 (Jun 2005)

[11] M. Hu, "Visual pattern recognition by moment invariants." IRE Trans. Inf. Theor. IT-8, 179-187 (1962)

[12] M. Mercimek, K.G.T.V. Mumcu, "Real object recognition using moment invariants." Sadhana, Academy Proceedings in Engineering Science 30(6), 765-775 (2005)

[13] D. Comaniciu, P. Meyer, "Mean shift: A robust approach toward feature space analysis." IEEE Transactions on Pattern Analysis and Machine Intelligence (PAMI) 24(5), 603-619 (2002)

[14] E.D. Demaine, M.L. Demaine, "Jigsaw puzzles, edge matching, and polyomino packing: Connections and complexity." Graphs and Combinatorics 23 (Supplement), 195-208 (2007), special issue on Computational Geometry and Graph Theory: The AkiyamaChvatalFestschrift.

[15] M. Barni, F. Bartolini, V. Cappellini, "Image processing for virtual restoration of artworks", Tools for Building Virtual Heritage, IEEE Multimedia, 34-37 (2000)

[16] I. Giakoumis, N. Nikolaidis, F. Pitas, "Digital image processing techniques for detection and removal of cracks in digitized paintings, IEEE Transactions on Image Processing 15 (1) 178-188, (2006)

[17] G. Spagnolo, F. Somma, "Virtual restoration of cracks in digitized image of paintings", Journal of physics. Conference series (vol. 249) (2010)

[18] A. Telea, "An image inpainting technique based on the Fast Marching Method", Journal of graphics tool (vol. 9), 25-36 (2003)

[19] M. Bertalmio, A. L. Bertozzi, G. Sapiro, "Navier-Stokes, fluid dynamics and Image and Video inpainting", Proc. ICCV 2001, 1335-1362 (2001).

[20] M. Oliveira, B. Bowen, R. McKenna, Y. Chang, "Fast digital image inpainting", Proc. VIIP 2001, 261-266 (2001)

[21] A. Gupta, V. Khandelwal, A. Gupta, M. C. Srivastava, "Image processing methods for the restoration of digitized paintings", Thammasat Int. J. Sc. Tech., Vol. 13, No.3, July-September 2008, 66-71 (2008)

[22] J. Serra. "Image Analysis and Mathematical Morphology", Volume 1. Academic Press (1984)

[23] J. Serra. "Image Analysis and Mathematical Morphology: Theoretical advances“, Volume 2. Academic Press (1988)

[24] G. Mercadante, "Una proposta di metodologia per il restauro virtuale delle screpolature dei dipinti" (A proposal of a methodology to virtual restoration of cracks in paintings), MSc thesis, Sapienza University of Rome, Information Engineering, Informatics, and Statistics (2012) 